\title{
Preoperative localization of suspicious parathyroid adenomas by assay of parathyroid hormone in needle aspirates
}

\author{
C Marcocci, S Mazzeo ${ }^{1}$, G Bruno-Bossio, A Picone, E Vignali, M Ciampi, P Viacava ${ }^{2}$, A G Naccarato ${ }^{2}$, P Miccoli ${ }^{3}$, \\ P Iacconi ${ }^{3}$ and A Pinchera \\ Dipartimento di Endocrinologia e Metabolismo, Ortopedia e Traumatologia, Medicina del Lavoro, Sezione di Endocrinologia, ${ }^{1}$ Dipartimento di Oncologia, \\ Divisione di Radiologia Diagnostica ed Interventistica, ${ }^{2}$ Dipartimento di Oncologia, Divisione di Anatomia Patologica and ${ }^{3}$ Dipartimento di Chirurgia, \\ Università di Pisa, Pisa, Italy \\ (Correspondence should be addressed to C Marcocci, Istituto di Endocrinologia, Università di Pisa, Via Paradisa 2, 56124, Pisa, Italy)
}

\begin{abstract}
Objective: To determine the usefulness of parathyroid hormone (PTH) measurement in needle aspirates of a suspicious neck mass to confirm its parathyroid nature in patients with primary hyperparathyroidism.

Methods: Thirty-three patients with surgically proved primary hyperparathyroidism were submitted to neck ultrasound (US), parathyroid scintigraphy, and assay of PTH in the aspirate (PTHa) of the suspicious cervical mass.

Results: Based on the results of neck US and parathyroid scintigraphy, patients were divided into two groups. Group 1: 16 patients (seven with nodular goiter) with concordant positive US and scintigraphic results. In all but one patient, PTHa was detectable and often markedly elevated $(>1000 \mathrm{pg}$ in 12 patients, between $292 \mathrm{pg}$ and $803 \mathrm{pg}$ in three patients and $53 \mathrm{pg}$ in one patient). The patient with undetectable PTHa had a small lower left parathyroid adenoma $(8 \times 8 \times 10 \mathrm{~mm})$. Group 2: 17 patients (12 with nodular goiter) with discordant US and scintigraphic results. PTHa established the parathyroid nature of the mass in 13 cases $(>1000 \mathrm{pg}$ in 8 patients, between $501 \mathrm{pg}$ and $953 \mathrm{pg}$ in three patients and 90 and $79 \mathrm{pg}$ in two patients): 11 of these had a suspected lesion by US examination but the scintigraphy results were negative; two had a mass that gave positive scintigraphy results but was of uncertain origin according to US; in both cases an intrathyroidal parathyroid adenoma was found. PTHa was undetectable in four cases (three with nodular goiter): all of these had equivocal US results, and three had positive scans and one a negative scan.

Conclusions: Assay of PTHa is a simple method and should be useful for confirming the parathyroid nature of a cervical mass in patients with discordant or non-diagnostic US and scintigraphic results.
\end{abstract}

European Journal of Endocrinology 139 72-77

\section{Introduction}

Primary hyperparathyroidism is a frequently diagnosed disease at the present time, particularly because of the routine measurement of serum calcium by multichannel serum analyser, by which many cases of asymptomatic or mildly symptomatic cases of hypercalcemia are discovered (1). A certain number of these patients require surgical treatment (1). There is considerable controversy about the indication for preoperative localization studies in primary neck exploration (2). Such studies are commonly regarded as unnecessary when parathyroid surgery is performed by an experienced surgeon, since a 90-95\% success rate has been reported in several series of patients undergoing initial surgical exploration (3). Although controversial, it has been suggested that preoperative localization in patients who have not undergone previous neck exploration can decrease operation time and morbidity, because only one side of the neck can be explored (see ref (4) for a review). Moreover, a positive preoperative localization investigation may give the patient increased confidence about the success of the operation and improve the success rate of lessexperienced surgeons (4-6). However, all investigators agree on the value of non-invasive and in some cases invasive techniques for patients with persistent or recurrent hyperparathyroidism after surgery $(1,4,6)$.

One of the problems that reduces the value of noninvasive localizing studies is the significant incidence $(15-20 \%)$ of false-positive results because of the difficulty of distinguishing the enlarged parathyroid gland from other cervical masses (4). This problem occurs particularly frequently when patients with hyperparathyroidism associated with nodular goiter are being evaluated (7), which is almost always the case in countries such as Italy and in Eastern Europe, where the prevalence of nodular goiter is extremely 
high because of iodine deficiency (8). In such cases confirmation of the parathyroid origin of a suspicious lesion could be obtained by measuring parathyroid hormone $(\mathrm{PTH})$ in the needle aspirate (PTHa) of the nodule (9-11).

In the present study we evaluated the usefulness of measuring PTHa of a cervical mass detected by ultrasound (US) and/or scintigraphy in a series of patients with primary hyperparathyroidism undergoing primary surgical neck exploration.

\section{Materials and methods}

\section{Patients}

The study was performed on 33 consecutive patients (eight males, 21 females, aged 14-80 years, mean 53 years) with recently diagnosed primary hyperparathyroidism and a suspicious enlarged cervical parathyroid gland detected by US and/or scintigraphy. All patients had hypercalcemia with a mean serum calcium of $11.7 \mathrm{mg} / \mathrm{dl}$ (range $10.5-18 \mathrm{mg} / \mathrm{dl}$ ) and increased levels of intact PTH (mean $222 \mathrm{pg} / \mathrm{ml}$, range $67-$ $1600 \mathrm{pg} / \mathrm{ml})$. Twenty patients $(60.6 \%)$ had an associated thyroid disease (16 had non-toxic nodular goiter, two papillary thyroid carcinoma, one Hashimoto's thyroiditis and one Graves' disease); all but the patient with Hashimoto's thyroiditis had goiter. All patients were submitted to parathyroid scintigraphy: thalliumtechnetium scintigraphy $(n=16)$, technetium-sestamibi scintigraphy $(n=13)$, or both $(n=4)$.

US-guided fine aspiration of the suspected parathyroid lesion was performed with the free-hand technique, using a 22 gauge needle (Spinal Needle: Terumo, Tokio, Japan). Two passes were performed on each lesion, without previous injection of saline into the mass. The aspirated material $(20-50 \mu \mathrm{l}$ depending upon the degree of blood contamination) was added to plastic vials containing $500 \mu \mathrm{l}$ PTH-free serum (standard $0^{\prime}$ of the PTH assay kit), centrifuged at 3000 r.p.m. for $10 \mathrm{~min}$ and the supernatant was stored at $-20^{\circ} \mathrm{C}$ until assayed. In patients with a typical US image of parathyroid adenoma (ovoidal, hypoechoic, see ref (12)), the aspiration was performed in the corresponding neck mass. When the US results were equivocal the selection of the mass to be aspirated was based on the scintigraphic results, when positive. In one patient (no. 31) aspiration was performed in a cervical mass of uncertain origin at US, which showed no uptake at scintigraphy.

Patients gave their informed consent to participate in the study, and the protocol was approved by the institutional review committee.

\section{Biochemical assays}

Serum calcium was measured by the $o$-cresolphthalein reaction (Merck, Darmstadt, Germany), and intact PTH by an IRMA (Nichols Institute, San Juan Capistrano, CA, USA). For PTH assay each sample was run in duplicate either undiluted or at 1:10 dilution in the PTH-free serum (standard 0). PTH values above $50 \mathrm{pg}$ in the aspirate were considered to be positive. In order to compare this value with circulating levels of PTH, the dilution factor should be taken into account, which ranged between 10 and 20 times, depending upon the degree of blood contamination. Therefore a $50 \mathrm{pg}$ content in the aspitate corresponds to a concentration of $1000-2000 \mathrm{pg} / \mathrm{ml}$ in the original sample before its dilution in PTH-free serum.

\section{Ultrasonography}

Sonographic examination was conducted by an experienced radiologist (SM) with high-resolution probes (7.5$10 \mathrm{MHz}$ ) mounted on commercially available scanners (AU 530 and AU 590 Asynchronous; Esaote Biomedica, Genova, Italy) (12). Each patient was placed in the supine position with neck hyperextended and then studied with transverse and longitudinal scans. In same cases the head was kept hyperextended and rotated to the left and right to obtain better visualization of the paraesophageal or paravertebral regions, where the ectopic gland can be located. A short vascular $7.5 \mathrm{MHz}$ linear probe (AL $33 \mathrm{~S})$, with a small skin contact area, was particularly useful in detecting parathyroid glands located in the thymus or upper mediastinum.

\section{Parathyroid scintigraphy}

The patient was positioned supine with the neck extended and immobilized. For thallium-technetium scintigraphy, $2 \mathrm{mCi}(74 \mathrm{MBq})$ of ${ }^{201}$ thallium (Tl) were injected i.v. and 20 images were acquired by a computerized $\gamma$ camera (400 and 3000; General Electric Medical System, Milwauke, WI, USA). While the patient remained supine, $5 \mathrm{mCi}(185 \mathrm{MBq}){ }^{99 \mathrm{~m}_{\mathrm{Tc}}-}$ pertechnectate (Tc) were injected and an additional 20 images were acquired. The final Tl-Tc images were obtained after correction for position, normalization for counting, and progressive subtraction of the Tc image from the Tl image. Image subtraction was carried out using standard General Electric software, without correction for cross-talk between $\mathrm{Tl}$ and Tc channels. The resulting images were then interpreted by two experienced nuclear physicians. An image obtained after the subtraction procedure is presumed to represent a parathyroid lesion. Cases with equivocal results were scored as negative.

${ }^{99} \mathrm{~m}$ Tc-Sestamibi (MIBI) scintigraphy was carried out by the procedure proposed by Taillefer et al. (13). Patients were injected i.v. with $20-25 \mathrm{mCi}$ (740-

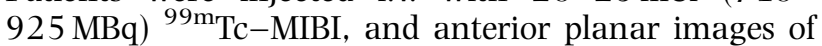
the neck and mediastinum were acquired with a pinhole low-energy collimator $30 \mathrm{~min}$ and $3 \mathrm{~h}$ after injection of the tracer. A positive result indicative of the presence 
Table 1 Results of US studies according to the absence or presence of goiter.

\begin{tabular}{lccc}
\hline & \multirow{2}{*}{$\begin{array}{c}\text { No. of } \\
\text { patients }\end{array}$} & Positive & Equivocal \\
\cline { 3 - 4 } & 33 & $28(85)$ & $5(15)$ \\
\hline Total & 19 & $16(80)$ & $4(20)$ \\
Presence of goiter & 14 & $12(92)$ & $1(8)$ \\
Absence of goiter & 14 & & \\
\hline
\end{tabular}

Values in parentheses are percentages.

of an abnormal parathyroid gland was established when focal uptake in the neck or mediastinum could be visualized in the later phase of imaging after the early uptake of tracer by the thyroid.

\section{Statistical analysis}

$\chi^{2}$ test was used to evaluate the relationships between imaging results and absence or presence of goiter.

\section{Results}

\section{Imaging procedures}

Twenty-eight (85\%) patients had a sonographically suspicious enlarged parathyroid gland; $5(15 \%)$ patients had equivocal results. Four of them had concomitant nodular goiter, and the neck mass discovered at US was believed to be of thyroid origin (Table 1).

Scintigraphic studies showed a positive result in 10 of $20(50 \%)$ patients evaluated with the ${ }^{201} \mathrm{Tl}-{ }^{99 \mathrm{~m}} \mathrm{Tc}$ technique, whereas using the ${ }^{99 \mathrm{~m}} \mathrm{Tc}-\mathrm{MIBI}$ technique a positive scan was observed in 13 of $17(76 \%)$ patients (Table 2). In the four patients in whom both techniques were performed, the results were concordant in three (two positive and one negative) and discordant in one (no. 26, positive with ${ }^{99 \mathrm{~m}} \mathrm{Tc}-\mathrm{MIBI}$ and negative with ${ }^{201} \mathrm{Tl}-{ }^{99 \mathrm{~m}} \mathrm{Tc}$ ). In goitrous patients, the sensitivity of the ${ }^{99 \mathrm{~m}} \mathrm{Tc}-\mathrm{MIBI}$ scintigraphy was significantly higher than that of the ${ }^{201} \mathrm{Tl}-{ }^{99 \mathrm{~m}} \mathrm{Tc}$ scintigraphy (positive results $10 / 12$ patients vs $5 / 11$ patients).

When US and scintigraphic results were compared, 16 patients (seven with goiter) had concordant positive results and 17 patients (12 with goiter) discordant results. Of the latter, 12 had positive US and negative scan, four positive scan and equivocal US study; only one patient had an equivocal US result with a negative parathyroid scan (no. 31). There was a statistically significant relationship between the results of US and scintigraphic studies (concordant or discordant) and the absence or presence of goiter $\left(\chi^{2}=21, P<0.0001\right)$.

In all but one patient (no. 31) the parathyroid adenoma was found at surgery in the location indicated by US and/or scintigraphy.

\section{PTH in the fine-needle aspirate}

Results for PTHa are reported in Table 3. In the 16 patients with concordant positive scintigraphic and echographic results, aspiration was performed to validate the measurement of РТHa as a procedure able to establish the parathyroid origin of the neck mass. In all but one patient, PTHa was detectable and often markedly increased $(>1000 \mathrm{pg}$ in 12 patients, ranged between $292 \mathrm{pg}$ and $803 \mathrm{pg}$ in three patients, and $53 \mathrm{pg}$ in one patient). The patient with undetectable PTHa had a small $(8 \times 8 \times 10 \mathrm{~mm})$ lower left parathyroid adenoma close to the inferior border of the left thyroid lobe, and it is conceivable that the negative result was due to erroneous sampling.

Table 4 shows the results in the 17 patients with discordant imaging results. As indicated, the PTH assay was clearly able to confirm the parathyroid origin of the neck mass in 13 of $17(76 \%)$ cases. In 11 of them, the US image suggested the parathyroid nature of the mass. In the remaining two patients (nos 6 and 8) with equivocal US results, the parathyroid adenoma was intrathyroidal. PTHa was $>500 \mathrm{pg}$ in 11 patients $(>1000 \mathrm{pg}$ in eight) and clearly detectable in two (79 pg and $90 \mathrm{pg}$ ).

PTHa was undetectable in four cases. One patient (no. 15) had a characteristic parathyroid lesion at US; the concomitant presence of nodular goiter had probably caused erroneous sampling. Three patients had uncertain US results. Two of them had thyroid nodules and a positive scan (nos 21 and 26) in the area where the adenoma was found at surgery. It is conceivable that in these patients a thyroid nodule

Table 2 Results of scintigraphic studies according to the absence or presence of goiter.

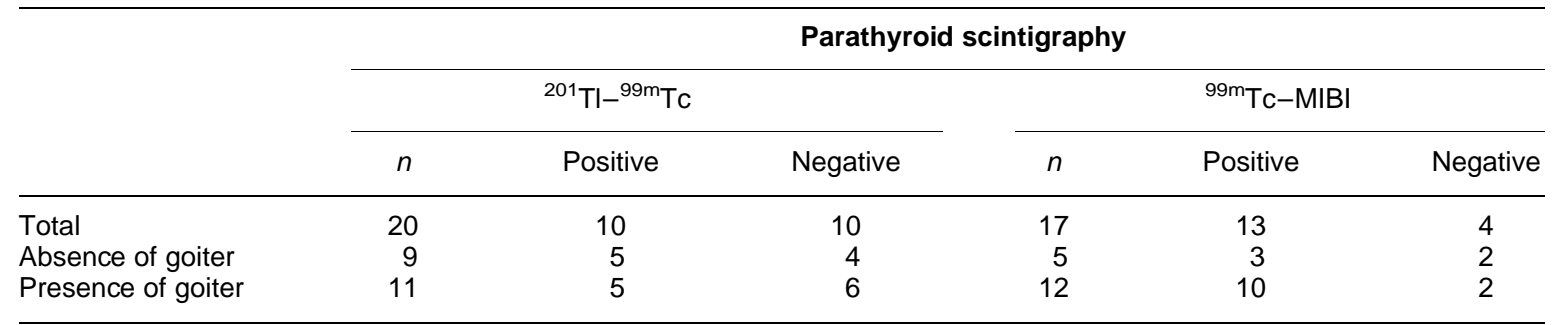

Patients with equivocal results were scored as negative. 
Table 3 Results of PTH assay according to US and scintigraphy (Scan) results and absence or presence of goiter.

\begin{tabular}{lllc}
\hline & & \multicolumn{2}{c}{ PTH assay } \\
\cline { 3 - 4 } US/Scan & Goiter & Positive & Negative \\
\hline \multirow{2}{*}{ Concordant $(n=16)$} & Absent $(n=9)$ & $9(100)$ & - \\
Discordant $(n=17)$ & Present $(n=7)$ & $6(86)$ & $1(14)$ \\
& Absent $(n=5)$ & $4(80)$ & $1(20)$ \\
& Present $(n=12)$ & $9(75)$ & $3(25)$ \\
\hline
\end{tabular}

The PTH assay in the aspirate was considered to be positive when $>50 \mathrm{pg}$.

Values in parentheses are percentages.

was aspirated; this hypothesis was confirmed by the finding of high thyroglobulin concentration in the aspirates (data not shown). One patient without goiter (no. 31) had a negative scan; the aspiration was performed in a small lesion which was on the contralateral side to that of the adenoma at surgery. In this case no thyroglobulin was detectable in the aspirate.

It is worth noting that four of the five patients with no PTH in the aspirate had nodular goiter, suggesting that the coexistence of thyroid enlargement could increase the likelihood of inadequate or erroneous sampling.

The aspiration procedure was well tolerated. Three patients had transient pain at the site of puncture and one had a minor local hemorrhage at surgery.

\section{Discussion}

Patients with primary hyperparathyroidism are often referred for localization studies before surgery, and US and/or parathyroid scintigraphy are usually performed as initial localizing studies, with a good overall diagnostic accuracy (12). However, the coexistence of a thyroid disease, particularly nodular goiter, may cause false-positive observations $(11,12,14)$. Over the past few years, major efforts have been put into developing new techniques to increase the sensitivity and accuracy of preoperative localization studies. The use of ${ }^{99 \mathrm{~m}} \mathrm{Tc}-$ MIBI has markedly improved scintigrapic results (4), but a few patients with surgically proved primary hyperparathyroidism still have false-negative results, particularly in the presence of thyroid diseases (12). In the field of US, increased accuracy has been obtained by integrating the gray-scale results with color Doppler study (14).

Despite this progress, a few patients may still have negative or equivocal results. Fine-needle aspiration for cytologic analysis often provides inadequate specimens, and in some instances the differential diagnosis between a thyroid and parathyroid nodule may be difficult (15-17). Measurement of PTH in the aspirate

Table 4 Clinical, biochemical and pathological data in the 17 patients with discordant US and scintigraphic (Scan) results.

\begin{tabular}{|c|c|c|c|c|c|c|c|}
\hline Patient & Goiter* & $\begin{array}{c}\text { Serum PTH } \\
(\mathrm{pg} / \mathrm{ml})\end{array}$ & USt & Scan† & $\begin{array}{l}\text { PTHa } \\
(\mathrm{pg}) \ddagger\end{array}$ & $\begin{array}{l}\text { Site of the } \\
\text { adenomat }\end{array}$ & $\begin{array}{l}\text { Size } \\
(\mathrm{mm})\end{array}$ \\
\hline 1 & + & 110 & $+(\mathrm{LL})$ & - & $>1000$ & LR & $25 \times 20 \times 6$ \\
\hline 2 & - & 187 & $+(\mathrm{LL})$ & - & $>1000$ & LL & $25 \times 15 \times 10$ \\
\hline 6 & + & 245 & $+1-$ & $+(\mathrm{LR})$ & 845 & LR & $30 \times 20 \times 20$ \\
\hline 8 & + & 660 & $+1-$ & $+(\mathrm{LL})$ & 541 & LL & $25 \times 20 \times 15$ \\
\hline 10 & + & 98 & $+(\mathrm{LR})$ & - & 953 & LR & $20 \times 10 \times 5$ \\
\hline 11 & - & 662 & $+(\mathrm{LL})$ & - & 1941 & $\mathrm{LL}$ & $17 \times 15 \times 15$ \\
\hline 12 & + & 72 & $+(\mathrm{LL})$ & - & $>1000$ & LL & $17 \times 8 \times 8$ \\
\hline 13 & - & 130 & $+(\mathrm{LL})$ & - & $>1000$ & LL & $20 \times 20 \times 13$ \\
\hline 15 & + & 83 & $+(U L)$ & - & $<8$ & UL & $20 \times 10 \times 5$ \\
\hline 17 & - & 224 & $+(\mathrm{LR})$ & - & 90 & LR & $25 \times 7 \times 5$ \\
\hline 19 & + & 102 & $+(\mathrm{LR})$ & - & 4375 & LR & $50 \times 20 \times 10$ \\
\hline 21 & + & 284 & $+1-$ & $+(U L)$ & $<8$ & UL & $35 \times 20 \times 15$ \\
\hline 26 & + & 72 & $+1-$ & $+(U L)$ & $<8$ & LL & $15 \times 10 \times 10$ \\
\hline 28 & + & 68 & $+(\mathrm{UR})$ & - & 1142 & UR & $10 \times 10 \times 8$ \\
\hline 29 & + & 107 & $+(\mathrm{LL})$ & - & $>1000$ & $\mathrm{LL}$ & $20 \times 10 \times 5$ \\
\hline 30 & + & 67 & $+(\mathrm{LR})$ & - & 79 & LR & $50 \times 10 \times 12$ \\
\hline 31 & - & 126 & $+1-(\mathrm{LL})$ & - & $<8$ & UR & $8 \times 10 \times 12$ \\
\hline
\end{tabular}

* + , presence of goiter; -, absence of goiter.

$\dagger+$, positive; +/-, equivocal; UR, upper right; LR, lower right; UL, upper left; LL, lower left.

$\ddagger$, the values represent the PTH content in the aspirated material; its volume ranged between 20 and $50 \mu$ depending upon blood contamination. 
of the suspicious parathyroid lesion to establish the parathyroid nature of the cervical mass has been proposed (9-11, 17-19). Previous studies have clearly shown the feasibility and accuracy of this procedure. Its use has been recommended for the evaluation of patients undergoing surgery after a previous negative neck exploration $(10,18)$.

In the present study we have evaluated the usefulness of measuring PTH levels in needle aspirates of 33 patients with primary hyperparathyroidism before surgery. All patients were examined before surgery by neck US and parathyroid scintigraphy. Sixteen showed concordant positive results. We performed needle aspiration of the suspicious parathyroid adenoma in these patients to validate the procedure; in all but one case, measurement of PTH confirmed the parathyroid nature of the neck mass. The false-negative result was obtained in a goitrous patient and was attributed to incorrect positioning of the needle because of the small size of the adenoma.

Seventeen patients $(51.5 \%)$ had discordant US and scan results. US was able to identify the suspicious (and surgically confirmed) parathyroid adenoma in four of five cases without goiter; all had a negative scan. On the other hand, in the presence of goiter, four of 12 patients had an equivocal cervical mass by US examination and a positive scan. This discrepancy probably resulted from the presence of a thyroid nodule in the area where a positive uptake was observed. In the remaining eight patients, US gave a positive result and scintigraphy a negative one.

Measurement of PTH in the needle aspirate allowed confirmation of the parathyroid nature of the neck mass in 13 of the 17 patients $(76.5 \%)$ with discordant US and scintigraphic results; three of the four patients with undetectable PTH in the aspirate also had nodular goiter. It is conceivable that, in these three patients, US misinterpreted the cervical lesion that was aspirated $(7,14)$; concentrations of thyroglobulin in the aspirate were high, indicating its thyroid nature. In the latter patient (no. 31) with negative parathyroid scan and equivocal US result, thyroglobulin was undetectable and the parathyroid adenoma was found on the contralateral side of the neck at surgery.

In three patients, PTH contents in the needle aspirates were below $250 \mathrm{pg}$ and similar to that of serum concentrations. In the evaluation of this result it is worth noting that the material obtained by aspiration was diluted at least 10 or 20 times before assay. Therefore clearly detectable PTH in the aspirate, even if it does not exceed the corresponding circulating level, can be considered to indicate the parathyroid nature of the aspirated mass.

Aspiration was well tolerated; only one patient had a small local hemorrhage which did not cause any significant trouble during surgery. One potential problem is related to the spilling or seeding of parathyroid cells along the needle track. Although this might in theory be a limiting factor, no case of recurrence of hyperparathyrodism has been reported in patients submitted to needle aspiration.

In the present study we evaluated only hyperparathyroid patients before a first neck exploration. The need for preoperative imaging studies in these patients remains controversial, and most authors agree that these procedures are unnecessary when the patient will be operated on by an experienced parathyroid surgeon (4). However, some patients are treated by surgeons with only limited experience in this field; in these cases, localization of the abnormal gland before surgery may improve the success rate $(4,5,20)$. Assay of PTH in the aspirate of the suspected lesion may be considered for this, in addition to other methods, such as computed tomography, magnetic resonance imaging and scintigraphy $(21,22)$.

However, there is no controversy about the need for preoperative localization studies before re-exploration in patients with persistent or recurrent hyperparathyroidism after surgery $(1,4,6)$. A combination of non-invasive techiques is generally used, and measurement of PTH in the aspirate has been proposed for cases with equivocal results before considering classical invasive techniques such as angiography and selective venous sampling (11). In this study, assay of PTH in the aspirate showed a sensitivity of $70 \%$ and a specificity of $100 \%$. Thus detection of PTH in the aspirate combined with US or CT images should give the exact anatomic location of the abnormal gland before surgery.

In conclusion, PTH assay in needle aspirates is simple and should be useful for establishing the parathyroid nature of a cervical mass. This procedure should be considered for patients with atypical US results (e.g. intrathyroidal nodules, coexistent goiter), and, because of its high specificity, it should be particularly useful for patients referred for re-exploration after unsuccessful parathyroid surgery. We do not recommend its use, or other localization studies, before the first exploratory operation, particularly if an experienced surgeon is available.

\section{References}

1 Silverberg SJ \& Bilezikian JP. Primary hyperparathyroidism: still evolving? Journal of Bone and Mineral Research 199712 856-862.

2 Doppman JL \& Miller DL. Localization of parathyroid tumors in patients with asymptomatic hyperparathyroidism and no previous surgery. Journal of Bone and Mineral Research 19916 S153-S159.

3 Norton JA, Brennan MF \& Wells SA. Surgical management of primary hyperparathyroidism. In The Parathyroids: Basic and Clinical Concepts, pp 531-552. Ed JP Bilezikian. New York: Raven Press.

4 Turton DB \& Miller DL. Recent advances in parathyroid imaging. Trends in Endocrinology and Metabolism 19967 163-168.

5 Freitas JA \& Freitas AE. Thyroid and parathyroid imaging. Seminars in Nuclear Medicine 199424 234-245.

6 Winzelberg GG. Imaging techniques for identifying parathyroid tumors. Trends in Endocrinology and Metabolism 19901 335-341. 
7 Karstrup S \& Hegedus L. Concomitant thyroid disease in hyperparathyroidism. European Journal of Radiology 19866 149-152.

8 Delange F \& Burgi H. Iodine deficiency disorders in Europe Bulletin of the World Health Organization 198967 317-325.

9 Doppman JL, Krudy AG, Markx SJ, Saxe A, Schneider P, Norton JA, Spiegel AM, Dowson RW, Schaaf M, Brennan ME, Schneider AB \& Aurbach GD. Aspiration of enlarged parathyroid glands for parathyroid hormone assay. Radiology 1983148 31-35.

10 Sacks BA, Pallotta JA, Cole A \& Hurwitz J. Diagnosis of parathyroid adenomas: efficacy of measuring parathormone levels in needle aspirates of cervical masses. American Journal of Roentgenology 1994163 1223-1226.

11 MacFarlane MP, Fraker DL, Shawker TH, Norton JA, Doppman JL, Chang RA, Skarulis MA, Marx SJ, Spiegel AM \& Alexander HR. Use of preoperative fine needle aspiration in patients undergoing reoperation for primary hyperparathyroidism. Surgery 1994116 959-965.

12 Mazzeo S, Caramella D, Lencioni R, Molea N, De Liperi A, Marcocci C, Miccoli P, Iacconi P, Bruno Bossio, Viacava P, Lazzeri E \& Bartolozzi C. Comparison among sonography, double-tracer subtraction and double-phase scintigraphy in the detection of parathyroid lesions. American Journal Roentgenology 1996166 1465-1470.

13 Taillefer R, Boucher Y, Potvin C \& Lambert R. Detection and localization of parathyroid adenomas in patients with hyperparathyroidism using the single radionuclide imaging procedure with technetium-99m-sesta-mibi (double-phase study) Journal of Nuclear Medicine 199233 1801-1807.

14 Mazzeo S, Caramella D, Lencioni R, Viacava P, De Liperi A, Naccarato AG, Armillotta N, Marcocci C, Miccoli P \& Bartolozzi C. Usefulness of echo-color Doppler in differentiating parathyroid lesions from other cervical masses. European Radiology 19977 90-95.
15 Tikkakoski T, Stenfors LE, Typpo T, Lohela $\mathrm{P} \&$ ApajaSarkkinen M. Parathyroid adenomas: pre-operative localization with ultrasound combined with fine-needle biopsy. Journal of Laryngology and Otology 1993107 543-545.

16 Solbiati L, Montali G, Croce F, Bellotti E, Giangrande A \& Ravetto C. Parathyroid tumors detected by fine-needle aspiration biopsy under ultrasonic guidance. Radiology $1983148793-797$.

17 Mincione D, Borrelli D, Cicchi P, Ipponi P \& Fiorini A. Fine needle aspiration cytology of parathyroid adenoma. Acta Cytologica 1986 $3065-69$.

18 Makarainen H, Raija T, Mayyi V K \& Pasi S. Cervical ultrasound combined with parathyroid hormone assay prior to parathyroid exploration. European Radiology 19922 194-198.

19 Bergenfelz A, Forsberg L, Hederstrom E \& Ahren B. Preoperative localization of enlarged parathyroid glands with ultrasonically guided fine needle aspiration for parathyroid hormone assay. Acta Radiologica $199132403-405$.

20 Satava RM, Beahrs OH \& Scholz DA. Success rate of cervical exploration for hyperparathyroidism. Archives of Surgery 1975 $110625-630$.

21 Rodriguez JM, Tezelman S, Siperstein AE, Duh AY, Higgins C, Morita E, Dowd CF \& Clark OH. Localization procedures in patients with persistent or recurrent hyperparathyroidism. Archives of Surgery 1994129 870-875.

22 McDermott VG, Mendez-Fernandez RJ, Meakem III TJ, Stolpen $\mathrm{AH}$, Spritzer $\mathrm{CH} \&$ Gefter WB. Preoperative MR imaging in hyperparathyroidsm: results and factors affecting parathyroid detection. American Journal of Roentgenology 1996166 705-710.

Received 23 October 1997

Accepted 16 March 1998 